\title{
HEINE-BOREL DOES NOT IMPLY THE FAN THEOREM
}

\author{
IEKE MOERDIJK
}

Introduction. This paper deals with locales and their spaces of points in intuitionistic analysis or, if you like, in (Grothendieck) toposes. One of the important aspects of the problem whether a certain locale has enough points is that it is directly related to the (constructive) completeness of a geometric theory. A useful exposition of this relationship may be found in [1], and we will assume that the reader is familiar with the general framework described in that paper.

We will consider four formal spaces, or locales, namely formal Cantor space $C$, formal Baire space $B$, the formal real line $R$, and the formal function space $R^{R}$ being the exponential in the category of locales (cf. [3]). The corresponding spaces of points will be denoted by $\mathrm{pt}(C), \mathrm{pt}(B), \mathrm{pt}(R)$ and $\mathrm{pt}\left(R^{R}\right)$. Classically, these locales all have enough points, of course, but constructively or in sheaves this may fail in each case. Let us recall some facts from [1]: the assertion that $C$ has enough points is equivalent to the compactness of the space of points $\operatorname{pt}(C)$, and is traditionally known in intuitionistic analysis as the Fan Theorem (FT). Similarly, the assertion that $B$ has enough points is equivalent to the principle of (monotone) Bar Induction (BI). The locale $R$ has enough points iff its space of points $\mathrm{pt}(R)$ is locally compact, i.e. the unit interval pt $[0,1] \subset \mathrm{pt}(R)$ is compact, which is of course known as the Heine-Borel Theorem (HB). The statement that $R^{R}$ has enough points, i.e. that there are "enough" continuous functions from $R$ to itself, does not have a well-established name. We will refer to it (not very imaginatively, I admit) as the principle (EF) of Enough Functions.

As is well known, $(\mathrm{BI}) \Rightarrow(\mathrm{FT}) \Rightarrow(\mathrm{HB})$. A possible way of explaining that (BI) implies (FT) is by observing that $B$ is homeomorphic to the exponential $C^{C}$, as has recently been pointed out by Hyland [3]. In the present context, therefore, the exponential $R^{R}$ is a natural object of study. Note that $(\mathrm{BI}) \Rightarrow(\mathrm{EF})$ since $R^{R}$ is countably presented, and hence a continuous image of $B$. The principle (FT) holds in every spatial topos, but $(\mathrm{BI})$ does not, so the implication $(\mathrm{BI}) \Rightarrow(\mathrm{FT})$ is not reversible (cf. [2]).

In $[1, \S 4.11]$, it was asked whether (HB) implies (FT). We will show that this is not the case by proving that $R$ has enough points in sheaves over the locale $K\left(\mathbf{R}^{2}\right)$ of coperfect open subsets of $\mathbf{R}^{2}$. Hyland has asked whether (BI) or (FT) follows from the assertion that $R^{R}$ has enough points. We will show that in the same topos of

Received October 5, 1982. 
sheaves over $K\left(\mathbf{R}^{2}\right)$, (EF) holds, thus answering Hyland's question negatively ( $\$ 2$ below). The converse implication $(\mathrm{FT}) \Rightarrow(\mathrm{EF})$ will also be seen to be false, but $(\mathrm{EF}) \Rightarrow(\mathrm{HB})$ is true $(\S 1)$.

Thus, our results complete the picture of valid implications in intuitionistic analysis, or in toposes, between the four statements (FT), (BI), (HB), and (EF): in the diagram below, the implications indicated are the only ones that hold.

$$
\begin{gathered}
(\mathrm{BI}) \Rightarrow(\mathrm{FT}) \\
\Downarrow \\
\Downarrow \\
(\mathrm{EF}) \Rightarrow(\mathrm{HB})
\end{gathered}
$$

§1. Internal locales. Let us begin with some conventions. There has been some confusion concerning the use of the terms locale, frame, space, etc. In this paper, the words locale and frame are used as in [1], and the elements of the frame corresponding to a locale $A$ will be called the opens of $A$. A space is a locale with enough points, or equivalently, a sober topological space. The product sign $\times$ will always denote the product in the category of locales. If $X$ and $Y$ are spaces their product in the category of topological spaces is denoted by $X \times{ }_{s} Y$; so $X \times{ }_{s} Y \cong \operatorname{pt}(X \times Y)$. As pointed out above, $C, B$ and $R$ all have enough points classically, and we will use $\mathbf{C}, \mathbf{B}$, and $\mathbf{R}$ to refer to the corresponding external spaces. $\mathbf{R}^{\mathbf{R}}$ refers to the external exponential, i.e. the space of continuous functions $\mathbf{R} \rightarrow \mathbf{R}$ with the compact-open topology.

If $A$ is an external locale, (internal) locales in $\operatorname{Sh}(A)$ are locales over $A$, i.e. continuous maps $X \rightarrow A$ of locales in the real world of sets, and internal continuous maps from $X$ to $Y$ correspond to external continuous maps over $A$. As is well known, formal Cantor space, formal Baire space, and the formal real line interpreted in $\operatorname{Sh}(A)$ are represented by the projections $\mathbf{C} \times A \rightarrow A, \mathbf{B} \times A \rightarrow A$, and $\mathbf{R} \times A \rightarrow A$. Since $R^{R}$ is defined as the exponential in the category of locales, it follows that its interpretation in $\operatorname{Sh}(A)$ is presented by $\mathbf{R}^{\mathbf{R}} \times A \rightarrow A$.

The following very useful observation is due to Hyland, and is also mentioned in [1].

1.1. Lemma. Let $X$ and $Y$ be sober spaces, i.e. locales with enough points. Then the internal locale $Y_{X}$ represented by the projection $Y \times X \rightarrow X$ has enough points in $\operatorname{Sh}(X)$ iff the locale product $Y \times X$ has enough points externally, i.e. coincides with $Y \times_{s} X$. (This happens for example if either $X$ or $Y$ is locally compact.)

Recall that a sublocale $A_{j}$ of a locale $A$ is called closed if $j$ is of the form (-) $\vee a$ for some open $a$ of $A$. It is easily seen that classically, a closed sublocale of a space is again a space. (This need not be true intuitionistically! Cf. 2.6 below.) We now immediately derive

1.2. THEOREM. (a) In spatial toposes, (EF) holds if and only if (BI) does.

(b) There exists a spatial topos in which (EF) fails, hence in particular (FT) does not imply (EF).

ProOF. (a) Note that in sets, $\mathbf{B}=\mathbf{N}^{\mathbf{N}}$ is homeomorphic to a closed subspace of $\mathbf{R}^{\mathbf{R}}$. Now suppose $X$ is any space such that $\operatorname{Sh}(X)$ satisfies (EF). Then by Lemma 1.1, $\mathbf{R}^{\mathbf{R}} \times X$ is spatial. But since the pullback of a closed sublocale is again closed, it follows from the remark above that $\mathbf{N}^{\mathbf{N}} \times X$ must be spatial, so by applying 1.1 again we find that $\operatorname{Sh}(X)$ satisfies (BI). Conversely, the implication $(\mathrm{BI}) \Rightarrow(\mathrm{EF})$ holds in any topos, as pointed out in the Introduction. 
(b) (BI) fails in sheaves over the space $\mathbf{Q}$ of rationals, but (FT) holds in any spatial topos (cf. [2]).

Let us observe that $R$ is a continuous image of $R^{R}$ : evaluation at a point of $R$ gives a surjective map $R^{R} \rightarrow R$ of locales. Hence (EF) implies (HB). Thus the only questions still open are whether (EF) implies (FT) and whether (HB) implies (FT). The first implication, and hence also the second, will be seen to be false in sheaves over $K\left(\mathbf{R}^{2}\right)$, to which we now turn.

\$2. The model over $K\left(\mathbf{R}^{2}\right)$. Let $j$ be the smallest nucleus ( $J$-operator, closureoperator) on $\mathcal{O}\left(\mathbf{R}^{2}\right)$ which identifies $U$ and $U \backslash\{t\}$, for each open $U$ and each point $t$. The resulting sublocale of $\mathbf{R}^{2}$ is denoted by $K\left(\mathbf{R}^{2}\right)$; its lattice of opens is precisely the lattice of complements of perfect closed subsets of $\mathbf{R}^{2}$. Obviously, $K\left(\mathbf{R}^{2}\right)$ cannot have any points. In [2], it was shown that (FT) fails in sheaves over $K\left(\mathbf{R}^{2}\right)$. We will show that (EF), and hence (HB), do hold over $K\left(\mathbf{R}^{2}\right)$, thus answering the remaining open questions mentioned above. To illustrate how small $K\left(\mathbf{R}^{2}\right)$ really is, we begin with the following observation.

2.1. Proposition. Let $A$ be any subset of $\mathbf{R}^{2}$ which does not contain a perfect closed set. Then the inclusion $K\left(\mathbf{R}^{2}\right) \hookrightarrow \mathbf{R}^{2}$ of locales factors through the inclusion $\mathbf{R}^{2} \backslash A \hookrightarrow \mathbf{R}^{2}$ of spaces, i.e. $K\left(\mathbf{R}^{2}\right)$ is a sublocale of $\mathbf{R}^{2} \backslash A$.

Proof. If $U$ is open in the subspace $\mathbf{R}^{2} \backslash A$, then $U=\operatorname{Int}(U \cup A) \backslash A$ (where the interior is taken in $\left.\mathbf{R}^{2}\right)$. For such a $U \in \mathcal{O}\left(\mathbf{R}^{2} \backslash A\right)$, we denote $\operatorname{Int}(U \cup A)$ by $\hat{U}$.

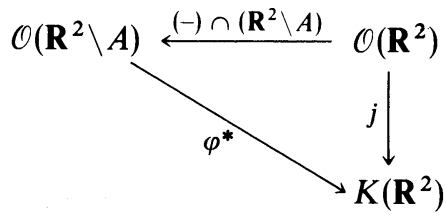

Now in the above diagram of frames, define $\varphi^{*}$ by $\varphi^{*}(U)=j(\hat{U})$. We claim that

(a) the diagram commutes, i.e. $j(V)=j(\operatorname{Int}(V \cup A))$ for each open $V \subseteq \mathbf{R}^{2}$, and

(b) $\varphi^{*}$ is a frame map, i.e. it preserves finite meets and arbitrary joins.

Proof of (a). Obviously, $j(V) \subseteq j(\operatorname{Int}(V \cup A))$. $\mathbf{R}^{2} \backslash j(V)$ is the largest perfect closed subset of $\mathbf{R}^{2} \backslash V$, and similarly for $\mathbf{R}^{2} \backslash j(\operatorname{Int}(V \cup A))$. So to prove that $j(\operatorname{Int}(V \cup A)) \subseteq j(V)$, it suffices to show that if $F$ is a perfect closed subset of $\mathbf{R}^{2} \backslash V$, we also have $F \subseteq \mathbf{R}^{2} \backslash \operatorname{Int}(V \cup A)=\overline{\mathbf{R}^{2} \backslash(V \cup A)}$. Now if $x$ is a point of such an $F$, let $W_{x}$ be an open neighbourhood of $x$, and let $C$ be a copy of the Cantor set, $C \subseteq W_{x} \cap F$. Then $C \cap V=\varnothing$, so $W_{x} \subseteq V \cup A$ would imply $C \subseteq A$, contradicting the assumption on $A$. Hence $W_{x} \nsubseteq V \cup A$, i.e. $x \in \overline{\mathbf{R}^{2} \backslash(V \cup A)}$.

PROOF OF (b). It is clear that $\varphi^{*}$ preserves the top and bottom elements, as well as binary intersections. Now let $\left\{U_{i} \mid i \in I\right\}$ be a collection of open subsets of $\mathbf{R}^{2} \backslash A$. We need to show that

$$
j\left(\bigcup_{i \in I} \operatorname{Int}\left(A \cup U_{i}\right)\right)=j\left(\operatorname{Int}\left(A \cup \bigcup_{i \in I} U_{i}\right)\right) .
$$

But by (a), it suffices to show that $A \cup \bigcup_{i \in I} \operatorname{Int}\left(A \cup U_{i}\right)=A \cup \operatorname{Int}\left(A \cup \bigcup_{i \in I} U_{i}\right)$, which is obviously true.

Thus, we have a map of locales $K\left(\mathbf{R}^{2}\right) \stackrel{\varphi}{\rightarrow} \mathbf{R}^{2} \backslash A$, giving the required factorization. 
We now turn to $\operatorname{Sh}\left(K\left(\mathbf{R}^{2}\right)\right)$. If $X$ is a space (externally), we obtain an internal locale represented by the projection $X \times K\left(\mathbf{R}^{2}\right) \rightarrow K\left(\mathbf{R}^{2}\right) . X \times K\left(\mathbf{R}^{2}\right)$ is a sublocale of $X \times \mathbf{R}^{2}$ (note that $X \times \mathbf{R}^{2}=X \times{ }_{s} \mathbf{R}^{2}$, since $\mathbf{R}^{2}$ is locally compact), and we have a pullback of locales

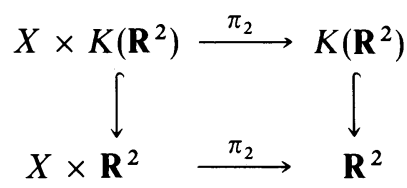

We write $1 \otimes j: \mathcal{O}\left(X \times \mathbf{R}^{2}\right) \rightarrow \mathcal{O}\left(X \times K\left(\mathbf{R}^{2}\right)\right)$ for the frame-map corresponding to the inclusion $X \times K\left(\mathbf{R}^{2}\right) \hookrightarrow X \times \mathbf{R}^{2}$. Thus each open of $X \times K\left(\mathbf{R}^{2}\right)$ is the form $1 \otimes j(W)$ for some subset $W$ of $X \times \mathbf{R}^{2}$, and we use $W$ as a name for this open of $X \times K\left(\mathbf{R}^{2}\right)$. Recall that the (global) opens of the internal locale $X \times K\left(\mathbf{R}^{2}\right) \rightarrow$ $K\left(\mathbf{R}^{2}\right)$ are just the opens of $X \times K\left(\mathbf{R}^{2}\right)$. The crucial part of the proof is the following lemma.

2.2. Lemma. Let $(X, d)$ be a complete metric space, and $W$ an open subset of $X \times \mathbf{R}^{2}$ such that $\operatorname{Sh}\left(K\left(\mathbf{R}^{2}\right)\right) \not W=\mathrm{T}$ when $W$ is regarded as an open of the internal locale $X \times K\left(\mathbf{R}^{2}\right) \rightarrow K\left(\mathbf{R}^{2}\right)$. Then there exist a copy $D \subseteq \mathbf{R}^{2}$ of the Cantor set and a continuous function $f: D \rightarrow X$ such that the graph of $f$ is disjoint from $W$. (T denotes the top element of any locale.)

Proof. It is notationally convenient to assume that complete metrics have been fixed in $X, \mathbf{R}^{2}$, and $X \times \mathbf{R}^{2}$, which are bounded by 1 , and such that $A \subseteq X$ and $B \subseteq \mathbf{R}^{2}, \operatorname{diam}(A \times B) \geq \max (\operatorname{diam}(A), \operatorname{diam}(B))$. By induction we will first define for each finite sequence $u \in 2^{<\mathrm{N}}$ a nonempty basic open subset $O_{u}=U_{u} \times V_{u}$ of $X \times \mathbf{R}^{2}$ such that for all sequences $u$ and $v$ :

1) $\bar{O}_{u} \subseteq O_{v}$ if $u$ extends $v, \bar{O}_{u} \cap \bar{O}_{v}=\varnothing$ if $u$ and $v$ are incompatible, and $\operatorname{diam}\left(O_{u}\right) \leq 2^{-1 \operatorname{th}(u)} ;$ and hence also

2) $\bar{V}_{u} \subseteq V_{v}$ if $u$ extends $v, \bar{V}_{u} \cap \bar{V}_{v}=\varnothing$ if $u$ and $v$ are incompatible, and $\operatorname{diam}\left(V_{u}\right) \leq 2^{-1 \operatorname{th}(u)} ;$ furthermore

3) $j\left(V_{u}\right) \not \neq O_{u} \leq W$ (where $j$ is the nucleus of 2.1).

For the case $u=\langle\rangle$, the empty sequence, we just take $U_{u}=X, V_{u}=\mathbf{R}^{2}$. For the induction step, the following observation is needed:

$\left.{ }^{*}\right)$ For any sentence $\varphi$, and any nonempty open $V \subseteq \mathbf{R}^{2}$, if $j(V) \not \neq \varphi$ then there are nonempty opens $V_{0}, V_{1} \subseteq V$ with $\bar{V}_{0} \cap \bar{V}_{1}=\varnothing$, such that $j\left(V_{0}\right) \not \varphi, j\left(V_{1}\right) \not \varphi$.

ProOF OF (*). If $j(V) \not \neq \varphi$, then $V-\llbracket \varphi \rrbracket$ must contain a perfect closed set, and hence a copy of the Cantor set $K$. Write $K$ as the disjoint sum of two copies $K_{0}$ and $K_{1}$ of the Cantor set, and let $V_{0}$ and $V_{1}$ be open neighbourhoods of $K_{0}$ and $K_{1}$ such that $\bar{V}_{0} \cap \bar{V}_{1}=\varnothing$. Then $K_{0} \subseteq V_{0}-\llbracket \varphi \rrbracket$, so $j\left(V_{0}\right) \not \llbracket \llbracket \varphi \rrbracket$, i.e. $j\left(V_{0}\right) \not \equiv \varphi$. Similarly $j\left(V_{1}\right) \not \models$. (The referee pointed out that this argument is somewhat simpler than my original proof of $(*)$.)

(*) being established, let us suppose that the $O_{u}$ have been defined for all sequences of length $n$, and pick one such sequence $u$. We will now define $\mathrm{O}_{u * 0}$ and $\mathrm{O}_{u * 1}$. Using $(*)$, we may choose two nonempty open sets $V_{0}, V_{1} \subseteq V_{u}$ such that $\bar{V}_{0} \cap \bar{V}_{1}=\varnothing$, and $j\left(V_{0}\right) \neq O_{u} \leq W, j\left(V_{1}\right) \not \equiv O_{u} \leq W$. That is, in $X \times K\left(\mathbf{R}^{2}\right)$, we have for each $i=0,1$,

$$
O_{u} \wedge \pi_{2}^{-1}\left(V_{i}\right) \not W \wedge O_{u} \wedge \pi_{2}^{-1}\left(V_{i}\right),
$$


or equivalently $U_{u} \times V_{i} \not \subset W$. Now keep $i$ fixed, and cover $U_{u} \times V_{i}$ in $X \times \mathbf{R}^{2}$ by open cubes $B_{i}^{\zeta}=U_{i}^{\zeta} \times V_{i}^{\zeta}$ of diameter $<2^{-(n+1)}$ ( $\zeta$ ranging over some indexing set), such that $\overline{B_{i}^{\zeta}} \subseteq U_{u} \times V_{i}$. Then the $B_{i}^{\zeta}$ also form a cover of $U_{u} \times V_{i}$ in $X \times K\left(\mathbf{R}^{2}\right)$, so for some $\zeta$, say $\zeta_{i}$, we must have that in $X \times K\left(\mathbf{R}^{2}\right), B_{i}^{\zeta} \not K W$. Now let $O_{u * i}=$ $B_{i}^{\zeta_{i}}=U_{i}^{\zeta_{i}} \times V_{i}^{\zeta_{i}}$, so $V_{u * i}=V_{i}^{\zeta_{i}} \subseteq V_{i}$. Then from the fact that $B_{i}^{\zeta_{i}} \not \leq W$ in $X \times K\left(\mathbf{R}^{2}\right)$ it follows that $O_{u * i} \not \leq W \cap \pi_{2}^{-1}\left(V_{u * i}\right)$ in $X \times K\left(\mathbf{R}^{2}\right)$, which just means that $j\left(V_{u * i}\right) \not \neq O_{u * i} \leq W$, so condition 3$)$ is satisfied. Conditions 1) and 2) are obvious.

We will now build our Cantor set $D$. Let us write $F=\left(X \times \mathbf{R}^{2}\right) \backslash W$, and $F_{u}=O_{u} \backslash W=\left(U_{u} \times V_{u}\right) \backslash W$. By condition 3), each $F_{u}$ is nonempty (in fact, from Proposition 2.1 we may even derive that $\pi_{2}\left(F_{u}\right)$ must contain a copy of the Cantor set). Hence we can choose a point $y_{u} \in F_{u}$ for each $u \in 2^{<\mathbf{N}}$. Write $x_{u}=\pi_{2}\left(y_{u}\right) \in V_{u}$. By condition 2), we find that for each $\alpha \in 2^{\mathbf{N}},\left\{x_{u}\right\}_{u \leq \alpha}$ is a Cauchy sequence, so it converges to a point $x_{\alpha} \in \mathbf{R}^{2}$. Note that also by 2 ), all the $x_{\alpha}$ must be different, since $x_{\alpha} \in \bar{V}_{u}$ if $u \leq \alpha$. Let $D=\left\{x_{\alpha} \mid \alpha \in 2^{\mathbf{N}}\right\}$. Then as a subspace of $\mathbf{R}^{2}, D$ is homeomorphic to the Cantor space. (The canonical map $\alpha \mapsto x_{\alpha}$ is a continuous bijection, hence a homeomorphism, from the Cantor space to $D$.)

By condition 1), each sequence $\left\{y_{u}\right\}_{u \leq \alpha}$ also is a Cauchy sequence. Hence it converges to a point $y_{\alpha}$, which must necessarily lie in the closed set $F$. Now let $g: D \rightarrow F \subseteq X \times \mathbf{R}^{2}$ be the function defined by $g\left(x_{\alpha}\right)=y_{\alpha}$. Then $g$ is continuous, and since $\pi_{2}\left(y_{\alpha}\right)=\lim _{u \leq \alpha} \pi_{2}\left(y_{u}\right)=\lim _{u \leq \alpha} x_{u}=x_{\alpha}, g$ must be of the form $(f, \mathrm{id})$, thus giving us the required continuous function $f: D \rightarrow X$.

Recall that a (Hausdorff) space $X$ is called an absolute neighbourhood retract (ANR) for a space $Y$ if for any closed subset $G \subseteq Y$, every continuous function $G \rightarrow X$ has a continuous extension over some open subset of $Y$ containing $G$.

2.3. THEOREM. If $X$ is a complete metrizable space and an ANR for $\mathbf{R}^{2}$, then the internal locale represented by $X \times K\left(\mathbf{R}^{2}\right) \rightarrow K\left(\mathbf{R}^{2}\right)$ has enough points in sheaves over $K\left(\mathbf{R}^{2}\right)$.

Proof. Let $W$ and $W^{\prime}$ be two open subsets of $X \times \mathbf{R}^{2}$ with $W \subseteq W^{\prime}$, such that if we regard them as names for internal opens of the locale $X \times K\left(\mathbf{R}^{2}\right) \rightarrow K\left(\mathbf{R}^{2}\right)$, then $\operatorname{Sh}\left(K\left(\mathbf{R}^{2}\right)\right) \vDash \operatorname{pt}\left(W^{\prime}\right) \subseteq \operatorname{pt}(W)$. We want to show that $\operatorname{Sh}\left(K\left(\mathbf{R}^{2}\right)\right) \vDash W^{\prime} \subseteq W$, and for this it suffices to show that for every cube $U \times V$ which is contained in $W^{\prime}$, $\operatorname{Sh}\left(K\left(\mathbf{R}^{2}\right)\right) \vDash U \times V \subseteq W$. If not, then we can apply Lemma 2.2 with $X$ replaced by $U$ and $\mathbf{R}^{2}$ replaced by $V$ to obtain a continuous function $g: D \rightarrow U$, where $D$ is perfect closed, such that for each $p \in D,(g(p), p) \notin W$. We can then extend $g$ to a continuous function $N \stackrel{f}{\rightarrow} U$ with open domain $N \subseteq V$, and the function $(f$, id $): N \rightarrow X \times \mathbf{R}^{2}$ then restricts to an internal point $q$ of $X \times K\left(\mathbf{R}^{2}\right) \rightarrow K\left(\mathbf{R}^{2}\right)$ defined over $j(N)$. Since $(f \text {, id })^{-1}(W) \cap D=\varnothing$, we cannot have $j(N) \vDash q \in W$, although we do have $j(N) \vDash q \in W^{\prime}$. This contradicts the fact that $\operatorname{Sh}\left(K\left(\mathbf{R}^{2}\right)\right) \vDash \operatorname{pt}\left(W^{\prime}\right) \subseteq \operatorname{pt}(W)$.

2.4. Corollary. (a) In $\operatorname{Sh}\left(K\left(\mathbf{R}^{2}\right)\right), R^{R}$ and $R$ have enough points, i.e. $\operatorname{Sh}\left(K\left(\mathbf{R}^{2}\right)\right)$ satisfies (EF) and (hence) (HB).

(b) (EF) does not imply (FT) (and hence neither does (HB)).

PROOF. As remarked at the beginning of this section, (b) follows from (a). (a) is immediate from the preceding theorem, since $\mathbf{R}^{\mathbf{R}}$ and $\mathbf{R}$ satisfy the hypothesis of 2.3 by Tietze's extension theorem for normal spaces.

The proof above does not use the full strength of Tietze's theorem. It is obvious that the same argument gives a more general version of 2.3 and 2.4: for example, we 
could replace $\mathbf{R}^{2}$ by any complete metric dense-in-itself space $Y$ and prove the analogous results for sheaves over $K(Y)$. I do not know any application of this more general fact.

2.5. Remark. It follows from Proposition 2.1 that if $W$ is an open subset of $X \times \mathbf{R}^{2}$ such that $\operatorname{Sh}\left(K\left(\mathbf{R}^{2}\right) \not \neq W=T, \pi_{2}\left(X \times \mathbf{R}^{2} \backslash W\right)\right.$ must contain a copy of the Cantor set. The converse is false, however. For example, let $C \subseteq \mathbf{R}^{2}$ be a fixed copy of the Cantor set, and let $X$ be the set $C$ with the discrete topology. Then if we let $W=X \times \mathbf{R}^{2} \backslash\{(x, x) \mid x \in C\}, W$ is open, but the image of $W$ under the quotient map of frames $1 \otimes j: \mathcal{O}\left(X \times \mathbf{R}^{2}\right) \rightarrow \mathcal{O}\left(X \times K\left(\mathbf{R}^{2}\right)\right)$ is the top-element $\mathrm{T}$; in other words, $\operatorname{Sh}\left(K\left(\mathbf{R}^{2}\right)\right) \models W=\mathrm{T}$. To see this, let $B_{x}=\{x\} \times \mathbf{R}^{2} \in O\left(X \times \mathbf{R}^{2}\right)$. Then for each $x \in C, \operatorname{Sh}\left(K\left(\mathbf{R}^{2}\right)\right) \models B_{x} \leq W$ (just omit the point $x$ from $R^{2}$ ); hence since the sets $B_{x}$ form an open cover of $X \times \mathbf{R}^{2}, \operatorname{Sh}\left(K\left(\mathbf{R}^{2}\right)\right) \vDash \mathrm{T}=\bigvee_{x} B_{x} \leq W$.

This explains why the Cantor set $D$ had to be constructed quite carefully in the proof of 2.2, and could not be obtained by just applying 2.1. However, if $X$ is compact, the converse does hold, and a considerably easier proof can be given of Lemma 2.2 for this case. Consequently, Theorem 2.3 can be proved much more easily for locally compact $X$. This covers the special case $\operatorname{Sh}\left(K\left(\mathbf{R}^{2}\right)\right) \vDash(\mathrm{HB})$ of Corollary 2.4, but does not apply to the case of $R^{R}$, of course.

Let me end this paper by drawing attention to another curious phenomenon in intuitionistic analysis illustrated by the model over $K\left(\mathbf{R}^{2}\right)$.

2.6. Corollary. (In localic toposes) A compact closed sublocale of a space need not be a space.

Proof. Look at $R$ and $C$ in $\operatorname{Sh}\left(K\left(\mathbf{R}^{2}\right)\right)$.

Acknowledgements. This paper was written in June 1982, when I was visiting the University of Cambridge. I would like to thank Martin Hyland, and the logic section of the Philosophy Department of the University of Amsterdam for making this visit possible. I am also grateful to Martin Hyland for drawing my attention to the questions that this paper answers.

\section{REFERENCES}

[1] M. P. Fourman and R. I. Grayson, Formal spaces, Proceedings of the Brouwer Centenary Conference, North-Holland, Amsterdam, 1982, pp. 107-122.

[2] M. P. Fourman and J. M. E. Hyland, Sheaf models for analysis, Applications of Sheaves (Proceedings of the Research Symposium, Durham, 1977), Lecture Notes in Mathematics, vol. 753, Springer-Verlag, Berlin, 1979, pp. 280-301.

[3] J. M. E. Hyland, Function spaces in the category of locales, Continuous Lattices (Proceedings of the Conference (Workshop IV), Bremen, 1979), Lecture Notes in Mathematics, vol. 871, Springer-Verlag, Berlin, 1981, pp. 264-281.

UNIVERSITY OF AMSTERDAM

AMSTERDAM, THE NETHERLANDS 